\title{
Thyroid Nodule Size as a Predictor of Malignancy in Follicular and Hurthle Neoplasms
}

\author{
Arunnit Boonrod ${ }^{1,2 *}$, Zeynettin Akkus ${ }^{2}$, M Regina Castro ${ }^{3}$, Atefeh Zeinodini ${ }^{2}$, \\ Kenneth Philbrick', Marius Stan ${ }^{3}$, Dana Erickson ${ }^{3}$, Bradley Erickson ${ }^{2}$
}

\begin{abstract}
Introduction: The management of follicular (FN) and Hurthle cell neoplasms (HCN) is often difficult because of the uncertainty of malignancy risk. We aimed to assess characteristics of benign and malignant follicular and Hurthle neoplasms based on their shape and size. Materials and methods: Patients with Follicular adenoma (FA) or carcinoma (FC) and Hurthle Cell adenoma (HCA) or carcinoma (HCC) who had preoperative ultrasonography were included. Demographic data were retrieved. Size and shape of the nodules were measured. Logistic regression analyses and odds ratios were performed. Results: A total of 115 nodules with 57 carcinomas and 58 adenomas were included. Logistic regression analysis shows that the nodule height and the patient age are predictors of malignancy ( $\mathrm{p}$-values $=0.001$ and 0.042 ). A cutoff value of nodule height $\geq 4 \mathrm{~cm}$. produces an odds ratio of 4.5 ( $\mathrm{p}$-value $=0.006$ ). An age $\geq 55$ year-old demonstrates an odds ratio of 2.4-3.6 ( $\mathrm{p}$-value $=0.03)$. Taller-than-wide shape was not statistically significant ( $\mathrm{p}$-value $=0.613$ ). Conclusion: FC and HCC are larger than FA and HCA in size, with a cutoff at $4 \mathrm{~cm}$. Increasing age increases the odds of malignancy with a cutoff at 55 year-old. Taller-than-wide shape is not a predictor of malignancy.
\end{abstract}

Keywords: Follicular neoplasm- hurthle cell neoplasm- size- taller-than-wide

Asian Pac J Cancer Prev, 22 (8), 2597-2602

\section{Introduction}

Follicular neoplasms (FN) and Hurthle cell neoplasms (HCN) represent $2-10 \%$ of thyroid nodules and they often cause challenges in managing patients with thyroid lesions. There is not yet a true consensus criteria for triaging these patients, since the main focus of thyroid cancer is mainly on the papillary thyroid carcinoma (PTC). For both FN and HCN, cytological features that are obtained with fine-needle aspiration biopsy (FNAB) cannot accurately differentiate benign lesions from malignant ones. Because vascular or capsular invasion is required to diagnose malignancy of these types of thyroid nodules, final pathology is essential (Carling and Udelsman, 2005; Mathur et al., 2014). Surgical treatment of these nodules ranges from hemithyroidectomy to total thyroidectomy. Even with data supporting adequacy of hemithyroidectomy in $74-96 \%$ of these cases, total thyroidectomy is often performed due to cost-effectiveness, risk of reoperation if a large nodule is found to be malignant and complications of reoperation (Melck et al., 2006; Wiseman et al., 2006; Corso et al., 2014). With better preoperative predictors, proper selection of the appropriate procedure would benefit patients in terms of postoperative complications, avoidance of reoperation, and overall quality of life
(Megwalu and Green, 2016; Kuba et al., 2017). Several ultrasound features, such as lack of a sonographic halo, hypoechoic appearance, predominantly solid contents, a heterogeneous echotexture, and the presence of calcifications, were reported to be predictors of follicular carcinomas (Sillery et al., 2010; Zhang and $\mathrm{Hu}, 2014$ ). In one study volume of FC was shown to be significantly higher than FA (Sillery et al., 2010). An increasing likelihood of follicular carcinoma (FC) and Hurthle cell carcinoma (HCC) in larger nodules has been reported (Méndez et al., 2008; Gulcelik et al., 2008; Choi et al., 2009; Sillery et al., 2010; Ibrahim et al., 2015; Arpana et al., 2018; Jin et al., 2018). However, there are not many articles had compared the size of FC and HCC directly to the size of follicular adenoma (FA) and Hurthle cell adenoma (HCA) and the results are discrepancy (Seo et al., 2009; Sillery et al., 2010; Zhang and Hu, 2014).

In this study, we evaluated the characteristics of FN and $\mathrm{HCN}$ based on their size, shape, and the patient data.

\section{Materials and Methods}

\section{Patients}

This study was approved by the local institutional review board with a waiver of informed consent. We

${ }^{1}$ Department of Radiology, Faculty of Medicine, Khon Kaen University, Khon Kaen, Thailand. ${ }^{2}$ Radiology Informatics Lab, Department of Radiology, Mayo Clinic, Rochester, MN, USA. ${ }^{3}$ Department of Endocrinology, Mayo Clinic, Rochester, MN USA. *For Correspondence: arunsi@kku.ac.th 
retrospectively reviewed pathology reports of patients who underwent thyroidectomy at our institution between January 2012 to December 2017 to identify patients with follicular or Hurthle cell adenomas or carcinomas in the final surgical pathology. Only subjects that have preoperative US with nodules can be identified surely in both transverse and longitudinal images, were included. Demographic data and thyroid stimulating hormone (TSH) level were recorded.

\section{US evaluation}

The nodules were measured in three axes by one neuroradiologist (AB). The first axis is the maximal dimension in the transverse image, the second is the maximal dimension perpendicular to the first dimension on the transverse image. These two axes are referred as width and depth, depending on the shape of the nodule. The last dimension (referred to as 'height') is the maximal dimension in the longitudinal image. (Tessler et al., 2017) (Figure 1)

A taller than wide shape appearance is determined based on the transverse image comparing the diameters parallel (tallness) and perpendicular (wideness) to the ultrasound beam (Tessler et al., 2017). From this description, we drew a bounding box around the nodule and calculated the ratio between the diameters in Y-axis and $\mathrm{X}$-axis of the nodule in transverse image such that the $\mathrm{X}$-axis was perpendicular to the US beam and the Y-axis was parallel (Figure 1). A Y/X axis ratio greater than 1 was classified as "beam-defined" taller than wide shape. We also calculated the ratio between depth and width from the three-axes measurement. These ratios would reflect the different angulation of the nodules which might simulate the various angulations on real time US. This parameter is referred to as the "diameter-defined" ratio in our study.

\section{Statistical analysis}

The SPSS v.22 software package was used for statistical analysis. Univariate binary logistic regression analysis was used for both categorical data (gender, TSH categories and shape) and continuous data (age, TSH value, width, perpendicular dimension and height). For each categorical data category, the predictor value and reference category were set. The independent variables which had P-value $<0.25$ were included in the next step for multivariable analysis. For multivariable analysis, forward logistic regression (LR) and backward LR methods were performed. The independent variables, which were statistically significant $(\mathrm{P}$-value $<0.05)$ in both methods, were then analysed by enter method LR to check the multicollinearity and interactions. The area under the curve (AUC) of receiver operator characteristic (ROC) curve was used for assessing the model performance. The beam-defined and diameter ratios for taller than wide shape are compared by paired t-test and correlation determination with Pearson's correlation.

\section{Results}

We found 556 patients with $\mathrm{FN}$ and $\mathrm{HN}$ as the final surgical pathology in our database. Of those, 397 had preoperative US available for our review. In patients with multiple nodules, the specific location of the nodule was not stated in 162 patients, causing uncertainty of nodule localization in US and thus such cases were excluded. One hundred twenty of the patients did not have both transverse and longitudinal images. This left a total of 115 patients that could be analyzed, including 58 benign (39 FA and 19 HCA) and 57 malignant (35 FC and 22 HCC).

The gender, age, width, depth, height and taller-than-wide appearance is found to be statistically significant $(\mathrm{P}$-values $=0.031,0.014,0.001,0.001$, $<0.001$, and 0.115 , respectively) based on the univariable binary logistic regression analysis of each independent variable (Table1). These variables were then analyzed by multivariable binary logistic regression analysis with forward LR and backward LR methods. In both methods, only height and age are considered statistically significant $(\mathrm{P}$-values $=0.001$ and 0.042 , respectively) $($ Table 2$)$. For visualization of $\mathrm{P}$-value trend of other variables, logistic regression analysis with enter method was performed and shown in Table 2. The area under the curve (AUC) of this model is 0.72 , representing a good model fit.

Nodule size was stratified by height and we found that increasing size increases the odds ratio (OR) of carcinoma. The categorized size reached statistical significance at size equal to or $>4 \mathrm{~cm}(\mathrm{P}$-value $=0.006, \mathrm{OR}=4.5)($ Table 3 and Figure 2).

Table 1. Descriptive Data and Univariable Logistic Regression Analysis

\begin{tabular}{|c|c|c|c|c|c|c|c|}
\hline & & Benign $(n=58)$ & Malignant $(n=57)$ & P-value & $\operatorname{Exp}(\mathrm{B})$ & \multicolumn{2}{|c|}{$95 \%$ C.I.for $\mathrm{EXP}(\mathrm{B})$} \\
\hline & & Mean (SD) or Count (\%) & Mean (SD) or Count (\%) & & & Lower & Upper \\
\hline \multicolumn{2}{|l|}{ Gender (Female) } & $41(70.7 \%)$ & $29(50.9 \%)$ & $0.031^{\mathrm{a}}$ & 0.429 & 0.199 & 0.925 \\
\hline \multicolumn{2}{|l|}{ Age } & $55.2(15)$ & $62.6(16)$ & $0.014^{\mathrm{a}}$ & 1.032 & 1.006 & 1.058 \\
\hline \multicolumn{2}{|c|}{ TSH Value (mIU/L) } & $2.28(1.45)$ & $2.48(1.53)$ & 0.585 & 1.094 & 0.792 & 1.513 \\
\hline \multirow[t]{3}{*}{ TSH level } & Low & $0 / 37(0 \%)$ & $1 / 32(3.1 \%)$ & 0.794 & 0 & 0 & \\
\hline & Normal & $30 / 37(81.1 \%)$ & $23 / 32(71.9 \%)$ & & & & \\
\hline & High & $7 / 37(18.9 \%)$ & $8 / 32(25 \%)$ & & & & \\
\hline Width (mm.) & & $22.7(10.8)$ & $32.9(18.9)$ & $0.001^{\mathrm{a}}$ & 1.048 & 1.018 & 1.079 \\
\hline Depth (mm.) & & $19.2(10.2)$ & $28.9(17.7)$ & $0.001^{\mathrm{a}}$ & 1.059 & 1.023 & 1.096 \\
\hline Height (mm.) & & $27.9(12.2)$ & $40.5(20.2)$ & $<0.001^{\mathrm{a}}$ & 1.047 & 1.021 & 1.074 \\
\hline Taller-than-wide & & $10(17.2 \%)$ & $17(29.8 \%)$ & $0.115^{\mathrm{a}}$ & 2.04 & 0.841 & 4.951 \\
\hline
\end{tabular}

a, Statistically significant for univariable analysis. 
Table 2. Multivariable Logistic Regression Analysis

\begin{tabular}{lcccccccc}
\hline & B & S.E. & Wald & df & Sig. & Exp(B) & \multicolumn{2}{c}{$95 \%$ C.I.for EXP(B) } \\
& & & & & & & Lower & Upper \\
\hline Age* & 0.027 & 0.013 & 4.148 & 1 & $0.042^{\mathrm{a}}$ & 1.028 & 1.001 & 1.055 \\
Gender** & -0.297 & 0.471 & 0.398 & 1 & 0.528 & 0.743 & 0.295 & 1.869 \\
Taller-than-wide** & 0.324 & 0.642 & 0.256 & 1 & 0.613 & 1.383 & 0.393 & 4.865 \\
Height* & 0.045 & 0.013 & 11.433 & 1 & $0.001^{\mathrm{a}}$ & 1.046 & 1.019 & 1.073 \\
Width** & -0.001 & 0.041 & 0 & 1 & 0.989 & 0.999 & 0.923 & 1.083 \\
Depth** & 0 & 0.039 & 0 & 1 & 0.998 & 1 & 0.925 & 1.08 \\
\hline
\end{tabular}

*, Variables analyzed by forward logistic regression (LR) and backward LR methods, ${ }^{* *}$, Variables analyzed by enter method, a Statistically significant for multivariable analysis.

Table 3. Odds Ratio of Tumor Size According to Height

\begin{tabular}{lcccc}
\hline Tumor size & OR & \multicolumn{2}{c}{$95 \%$ C.I. } \\
& & P-value & Lower & Upper \\
\hline $1-1.9 \mathrm{~cm}$. & 1 & 0.042 & & \\
$2-2.9 \mathrm{~cm}$. & 2.013 & 0.195 & 0.698 & 5.806 \\
$3-3.9 \mathrm{~cm}$. & 1.687 & 0.446 & 0.439 & 6.489 \\
$\geq 4 \mathrm{~cm}$. & 4.5 & $0.006^{\mathrm{a}}$ & 1.549 & 13.07 \\
\hline
\end{tabular}

OR, Odds ratio; a, Statistical significant difference at size equal to or more than $4 \mathrm{~cm}$.

Stratified age groups showed increase OR for carcinoma with increase age. The optimal cutoff value is at 55 year-old $(\mathrm{OR}=2.4-3.6, \mathrm{p}$-value $=0.03)$. (Table 4$)$.

Pearson's correlation demonstrated strong, positive correlation between beam-defined and diameter-defined ratios $(r=0.768, p$-value $=0.01)$ (Figure 3$)$. The mean (SD) of beam-defined and diameter-defined ratios for nodule shape of all data were $0.85( \pm 0.21)$ and 0.88
Table 4. Odds Ratio of Age

\begin{tabular}{lcccc}
\hline Age (year) & OR & p-value & \multicolumn{2}{c}{$95 \%$ C.I. } \\
& & & Lower & Upper \\
\hline$\leq 55$ & 1 & 0.032 & & \\
$56-75$ & 2.431 & $0.033^{\text {a }}$ & 1.072 & 5.512 \\
$\geq 76$ & 3.647 & $0.027^{\text {a }}$ & 1.161 & 11.457 \\
\hline OR, Odds ratio; ${ }^{\text {a }}$, Statistical significant difference at age more than
\end{tabular}

55 year-old.

$( \pm 0.26)$, respectively ( $\mathrm{p}$-value $=0.051)$. When excluded the equivocal ratios (ratios range from 0.9-1.1), the mean (SD) were $0.78( \pm 0.23)$ and $0.81( \pm 0.27)$, respectively $(\mathrm{p}$-value $=0.118)$.

\section{Discussion}

Our study showed that size and age are independent predictors of malignancy in follicular and Hurthle cell

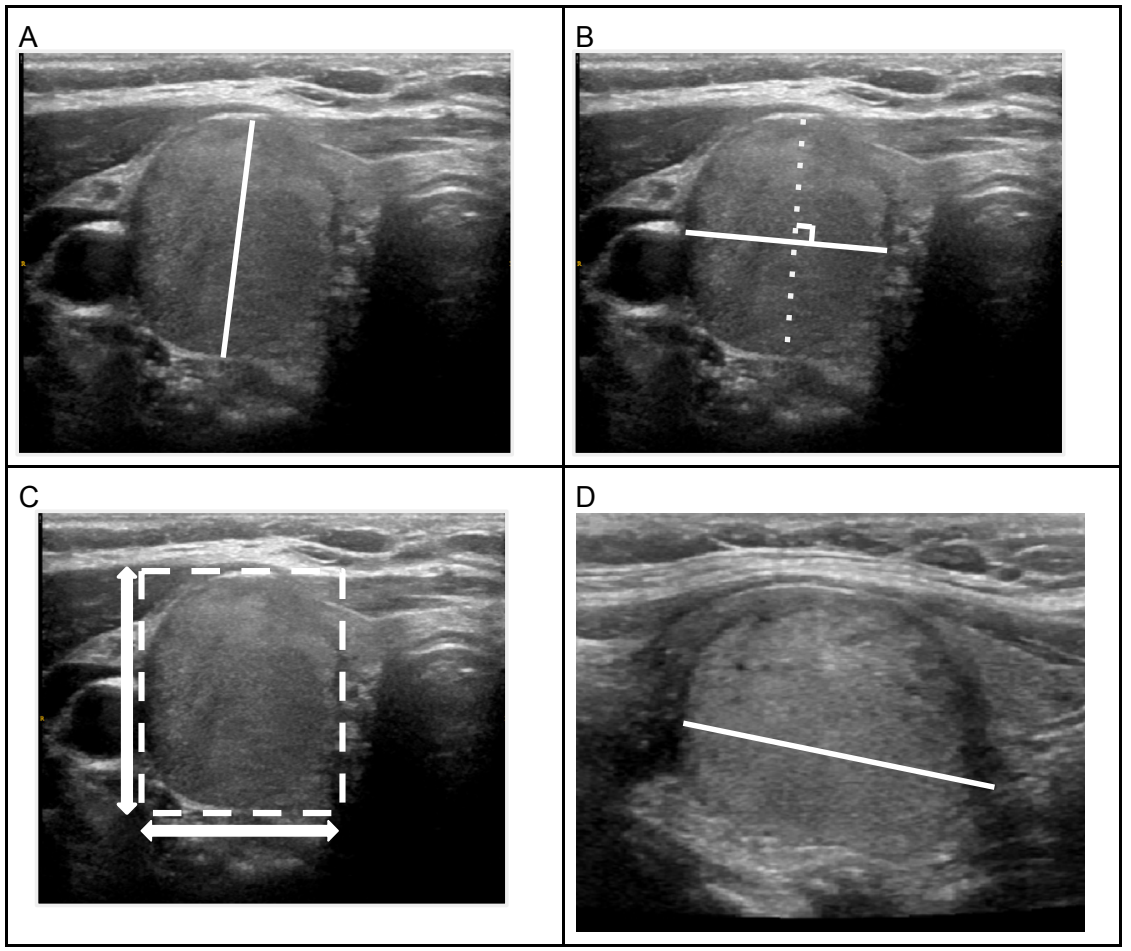

Figure 1. Transverse (A, B and C) and longitudinal (D) US images of an isoechoic solid thyroid nodule demonstrating the measurement method (A, B and D; depth, width and height diameters). Bounding box is drawn around the nodule (C) and the ratio between $Y$ and $X$ axes represents the shape of nodule. The shape of this nodule is taller-than-wide. The final pathology is a follicular adenoma. 


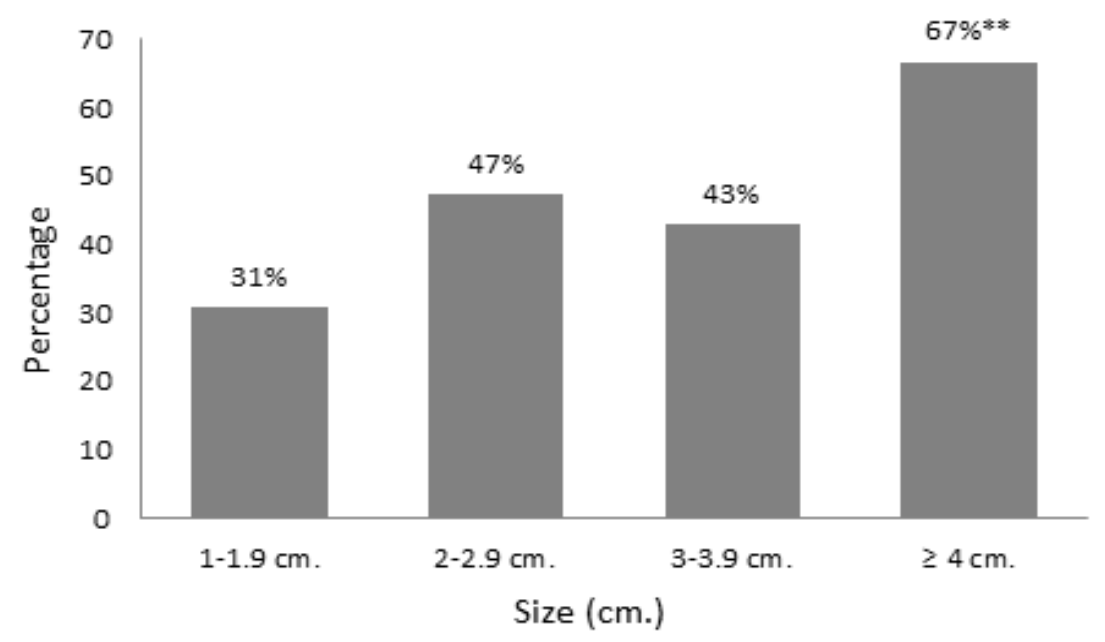

Figure 2. Percentage of Malignancy According to Nodule Height. **P-value $=0.006$

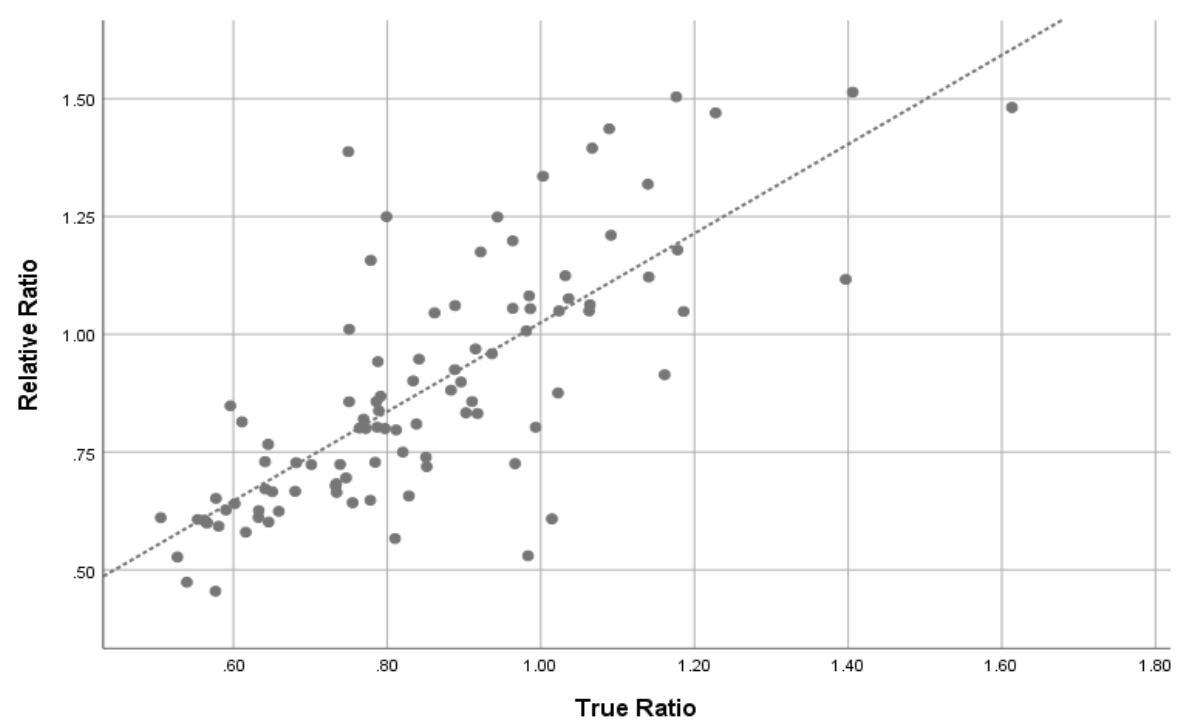

Figure 3. Scatter Plot of Beam-Defined and Diameter-defined Ratios. $(r=0.768$, $p$-value $=0.01)$

tumors. FC and $\mathrm{HC}$ are significantly larger than adenomas with the optimal cutoff at size $\geq 4 \mathrm{~cm}(\mathrm{OR}=4.5$, $\mathrm{p}$-value $=0.006)$. Several studies mentioned that FC and HCC tend to be larger in size as compared to other types of malignancy but they did not compare them to their benign counterparts (Méndez et al., 2008; Gulcelik et al., 2008; Choi et al., 2009; Ibrahim et al., 2015; Jin et al., 2018). Increasing tumor volume was also reported to increase the risk of malignancy in FN (Sillery et al., 2010). Previous studies on FN cytology reported that size was not a predictor of malignancy, however these studies compared final pathology of malignancy with final benign pathology without subclassification of a specific tumor subtype; e.i. Follicular and papillary carcinomas were considered all together as malignancy (Méndez et al., 2008; Gulcelik et al., 2008; Choi et al., 2009; Parikh et al., 2013; Ibrahim et al., 2015). When it comes to the size, measurement method is crucial. Our study is one of the few studies describing the method of measurement, which we did according to a standard system (Tessler et al., 2017).

Even though increasing age unsurprisingly increases risk of malignancy, we showed the odds ratio of each age group, which could be of additional value for triaging patients based on this basic parameter. We found an optimal cutoff age at 55 year-old $(\mathrm{OR}=2.4-3.6$, $\mathrm{p}$-value $=0.03$ )

To the best of our knowledge, our study is the first showing that taller-than-wide shape is not an independent predictor for malignancy in FN and HCN. Taller-thanwide shape has been well accepted to be an important malignant US feature in thyroid cancer. However, most studies on thyroid cancer are weighted toward findings of papillary carcinoma which has different nature both clinically and histologically (Raparia et al., 2009; Yoon et al., 2010; Castro et al., 2011; Ren et al., 2015; Espinosa De Ycaza et al., 2016; Tessler et al., 2017; Yang and Fried, 2018). A previous study using CT comparing shape of $\mathrm{FN}$ and nodular hyperplasia found that taller-than-wide shape is significantly more common in FN, irrespective of adenoma or carcinoma (Lee et al., 2016). Several studies have reported that follicular variant of PTC has significantly less taller-than-wide appearance as compared to conventional PTC (Kim et al., 2009; Anuradha et 
al., 2016; Jeon et al., 2016). Interobserver agreement of taller than wide shape ranges from fair to almost perfect (kappa ranges from 0.282-1) in previous studies. (Chandramohan et al., 2016; Tessler et al., 2017; Grani et al., 2018; Phuttharak et al., 2019; Pang et al., 2019). We believe this variation occurs because of variability in the position of the US probe in real time scanning. We found a near-significant difference between the beam-defined and diameter-defined ratios on the entire data ( $p$-value $=0.051$ ) but after excluding the equivocal ratios (ratios range from 0.9-1.1), the difference was not significant $(p$-value $=0.118)$. Angulations of the probe would not have a serious effect on nodules that are strikingly taller than wide. However, for nodules that are equivocal, the different angulation will affect the ratio; hence affect the overall shape. Inspection alone without measurement will cause discordant comments on the same nodule.

Limitations of this study is that the data is from a single institution and it was collected retrospectively. A large number of patients were excluded due to the incompleteness of data (e.g. both longitudinal and perpendicular views). Nonetheless, our study has one of the highest numbers of follicular and Hurthle cell neoplastic pathology with almost balanced number of adenomas and carcinomas.

In conclusion, FC and $\mathrm{HCC}$ are significantly larger than FA and HCA with a significant cutoff value at $4 \mathrm{~cm}$. Increasing age groups show increasing odds ratios of malignancy. Our study suggests that taller-than-wide shape is not a predictor for carcinoma in this group of patients, in contrast to PTC. We believe that this knowledge will be helpful in more accurately assessing the patient's risk of malignancy thus better guiding subsequent management.

\section{Author Contribution Statement}

A.B. and Z.A. conceived of the presented idea. A.B. developed the theory and performed the computations. A.Z and K.P. contributed to sample preparation. C.D. and D.E. verified the analytical methods. M.R.C, M.S., D.E and B.E. supervised the findings of this work. All authors discussed the results and contributed to the final manuscript.

\section{Acknowledgements}

This study was approved by the Mayo Clinic institutional review board with a waiver of informed consent.

This study is not a part of an approved student thesis.

\section{Conflict of interest}

All authors certify that they have no conflict of interest.

\section{References}

Anuradha C, Manipadam M, Asha H, et al (2016). Can new ultrasound signs help in identifying follicular variant of papillary carcinoma of thyroid? - A Pilot Study. Ultrasound Int Open, 2, 47-53

Arpana, Panta OB, Gurung G, Pradhan S (2018). Ultrasound findings in thyroid nodules: A Radio-Cytopathologic Correlation. J Med Ultrasound, 26, 90-3.

Carling T, Udelsman R (2005). Follicular neoplasms of the thyroid: What to Recommend. Thyroid, 15, 583-7.

Castro MR, Espiritu RP, Bahn RS, et al (2011). Predictors of malignancy in patients with cytologically suspicious thyroid nodules. Thyroid, 21, 1191-8.

Chandramohan A, Khurana A, Pushpa BT, et al (2016). Is TIRADS a practical and accurate system for use in daily clinical practice?. Indian J Radiol Imaging, 26, 145-52.

Choi YJ, Yun JS, Kim DH (2009). Clinical and ultrasound features of cytology diagnosed follicular neoplasm. Endocrine J, 56, 383-9.

Corso C, Gomez X, Sanabria A, et al (2014). Total thyroidectomy versus hemithyroidectomy for patients with follicular neoplasm. A cost-utility analysis. In J Surg, 12, 837-42.

Espinosa De Ycaza AE, Lowe KM, Dean DS, et al (2016). Risk of malignancy in thyroid nodules with non-diagnostic fineneedle aspiration: A Retrospective Cohort Study. Thyroid, 26, 1598-1604

Grani G, Lamartina L, Cantisani V, et al (2018). Interobserver agreement of various thyroid imaging reporting and data systems. Endocr Connect, 7, 1-7

Gulcelik NE, Gulcelik MA, Kuru B (2008). Risk of malignancy in patients with follicular neoplasm: predictive value of clinical and ultrasonographic features. Arch Otolaryngol Head Neck Surg, 134, 1312-5.

Ibrahim Y, Mohamed SEH, Deniwar A, et al (2015). The impact of thyroid nodule size on the risk of malignancy in follicular neoplasms. Anticancer Res, 35, 1635-9.

Jeon EJ, Jeong YJ, Park SH, et al (2016). Ultrasonographic characteristics of the follicular variant papillary thyroid cancer according to the tumor size. J Korean Med Sci, 31, 397-402

Jin W-X, Ye D-R, Sun Y-H, et al (2018). Prediction of central lymph node metastasis in papillary thyroid microcarcinoma according to clinicopathologic factors and thyroid nodule sonographic features: a case-control study. Cancer Manag Res, 10, 3237-43.

Kim DS, Kim J-H, Na DG, et al (2009). Sonographic features of follicular variant papillary thyroid carcinomas in comparison with conventional papillary thyroid carcinomas. J Ultrasound Med, 28, 1685-92.

Kuba S, Yamanouchi K, Hayashida N, et al (2017). Total thyroidectomy versus thyroid lobectomy for papillary thyroid cancer: Comparative analysis after propensity score matching: A multicenter study. Int J Surg, 38, 143-8.

Lee KH, Kim DW, Baek JW, et al (2016). Comparison of computed tomography features between follicular neoplasm and nodular hyperplasia. Cancer Imaging, 16, 31 .

Mathur A, Olson MT, Zeiger MA (2014) Follicular lesions of the thyroid. Surg Clin North Am, 94, 499-513.

Megwalu UC, Green RW (2016). Total thyroidectomy versus lobectomy for the treatment of follicular thyroid microcarcinoma. Anticancer Res, 36, 2899-2902.

Melck A, Bugis S, Baliski C, et al (2006). Hemithyroidectomy: the preferred initial surgical approach for management of Hurthle cell neoplasm. Am J Surg, 191, 593-7.

Méndez W, Rodgers SE, Lew JI, et al (2008). Role of surgeonperformed ultrasound in predicting malignancy in patients with indeterminate thyroid nodules. Ann Surg Oncol, 15, 2487-92

Pang Z, Margolis M, Menezes RJ, et al (2019). Diagnostic performance of 2015 American Thyroid Association guidelines and inter-observer variability in assigning risk category. Eur J Radiol Open, 6, 122-7.

Parikh PP, Allan BJ, Lew JI (2013). Surgeon-performed 
ultrasound predictors of malignancy in patients with Hürthle cell neoplasms of the thyroid. J Surg Res, 184, 247-52.

Phuttharak W, Boonrod A, Klungboonkrong V, Witsawapaisan $\mathrm{T}$ (2019). Interrater reliability of various thyroid imaging reporting and data system (TIRADS) classifications for differentiating benign from malignant thyroid nodules. Asian Pac J Cancer Prev, 20, 1283-8.

Raparia K, Min SK, Mody DR, et al (2009). Clinical outcomes for "suspicious" category in thyroid fine-needle aspiration biopsy: Patient's sex and nodule size are possible predictors of malignancy. Arch Pathol Lab Med, 133, 787-90.

Ren J, Liu B, Zhang L-L, et al (2015). A taller-than-wide shape is a good predictor of papillary thyroid carcinoma in small solid nodules. J Ultrasound Med, 34, 19-26

Seo HS, Lee DH, Park SH, et al (2009). Thyroid follicular neoplasms: can sonography distinguish between adenomas and carcinomas?. J Clin Ultrasound, 37, 493-500

Sillery JC, Reading CC, Charboneau JW, et al (2010). Thyroid follicular carcinoma: sonographic features of 50 cases. AJR Am J Roentgenol, 194, 44-54.

Tessler FN, Middleton WD, Grant EG, et al (2017). ACR thyroid imaging, reporting and data system (TI-RADS): white paper of the ACR TI-RADS committee. $J$ Am Coll Radiol, 14, 587-95.

Wiseman SM, BaliskiC, Irvine R, et al (2006). Hemithyroidectomy: The optimal initial surgical approach for individuals undergoing surgery for a cytological diagnosis of follicular neoplasm. Ann Surg Oncol, 13, 425-32.

Yang GCH, Fried KO (2018). Pathologic basis of the sonographic differences between thyroid cancer and noninvasive follicular thyroid neoplasm with papillary-like nuclear features. Ultrasonography, 37, 157-63.

Yoon SJ, Yoon DY, Chang SK, et al (2010). Taller-than-wide sign" of thyroid malignancy: comparison between ultrasound and CT. AJR Am J Roentgenol, 194, 420-4.

Zhang J-Z, Hu B (2014). Sonographic features of thyroid follicular carcinoma in comparison with thyroid follicular adenoma. J Ultrasound Med, 33, 221-7.

This work is licensed under a Creative Commons AttributionNon Commercial 4.0 International License. 